TRANSACTIONS OF THE

AMERICAN MATHEMATICAL SOCIETY

Volume 348, Number 1, January 1996

\title{
FAILURE OF NORMALITY IN THE BOX PRODUCT OF UNCOUNTABLY MANY REAL LINES
}

\author{
L. BRIAN LAWRENCE
}

Dedicated to Mary Ellen Rudin and A. H. Stone

\begin{abstract}
We prove in ZFC that the box product of $\omega_{1}$ many copies of $\omega+1$ is neither normal nor collectionwise Hausdorff. As an addendum to the proof, we show that if the cardinality of the continuum is $2^{\omega_{1}}$, then these properties also fail in the closed subspace consisting of all functions which assume the value $\omega$ on all but countably many indices.
\end{abstract}

\section{INTRODUCTION}

History. More than thirty years have passed since A. H. Stone first raised the issue of normality and paracompactness in box products (1964, [Kn]), and more than twenty years since Mary Ellen Rudin achieved the first breakthrough (1972, $\left.\left[\mathrm{Ru}_{1}\right]\right)$. A box product is a topological space which takes a cartesian product of spaces for the point-set, and takes an arbitrary cartesian product of open subsets for a base element; note that in contrast to the Tychonoff topology of pointwise convergence, each factor of a basic open set is permitted to be a proper subset of the factor space. Suppose $\Lambda$ is a separable metric space and $\nu$ is a cardinal number. Let $\square^{\nu}(\Lambda)$ denote the box product where $\nu$ is the index set and each factor is $\Lambda\left({ }^{\nu} \Lambda\right.$ denotes the point-set consisting of all functions from $\nu$ into $\Lambda)$. Is $\square^{\nu}(\Lambda)$ normal (paracompact)? Rudin showed in the paper cited above that the answer is yes (for paracompact) if $\nu=\omega, \Lambda$ is locally compact, and the Continuum Hypothesis $(\mathrm{CH})$ holds. What happens if the index set is uncountable? This aspect of Stone's problem was mentioned in particular by Rudin in her Lectures on Set-Theoretic Topology $\left(1975,\left[\mathrm{Ru}_{2}\right]\right)$. The purpose of the current paper is to show in ZFC that $\square^{\omega_{1}}(\omega+1)$ is neither normal nor collectionwise Hausdorff (a space has the latter property provided that every closed discrete subset $D$ can be separated, i.e., there is a 1-1 correspondence between $D$ and a pairwise disjoint collection of open sets such that each point of $D$ belongs to the corresponding set; and a subset $D$ is discrete provided that each $x \in D$ has a neighborhood disjoint from $D \backslash\{x\})$. Note that $\square^{\omega_{1}}(\omega+1)$ is homeomorphic to a closed subspace of $\square^{\nu}(\Lambda)$ whenever $\nu \geq \omega_{1}$ and $\Lambda$ is a nondiscrete metric space.

Received by the editors November 22, 1991 and, in revised form, October 31, 1994.

1991 Mathematics Subject Classification. Primary 54D18; Secondary 54A35, 54B10, 54B20.

Key words and phrases. Box product, normal, paracompact, collectionwise Hausdorff, continuum hypothesis.

An abstract of this paper was presented at the Summer Topology Conference in Honor of Mary Ellen Rudin, University of Wisconsin, Madison, June, 1991.

(C)1996 American Mathematical Society 
Organization of the paper. We will need to put in place some fairly complicated machinery, so we begin by introducing just enough notation $(\S 1)$ to give an outline of the proof $(\S 2)$. The outline explains both the overall framework and the roles played by the various individual concepts; it is not intended as casual reading but as an important first step to understanding the full details of the proof.

As an additional aid to the reader, the section breakdown is designed to focus attention on each of the major ideas individually.

The proof of the main theorem can be modified to show that if $c=2^{\omega_{1}}$ (as usual, $c$ denotes $2^{\omega}$ ), then, in the following closed subspace, normality and the collectionwise Hausdorff property also fail: $\left\{f \in \square^{\omega_{1}}(\omega+1):\left|\left\{\alpha \in \omega_{1}: f(\alpha)<\omega\right\}\right| \leq \omega\right\}$ (the vertical bars denote the cardinality operator). Let $P=\left\{u \subseteq \omega_{1}:|u|=\omega_{1}\right\}$ and let $Q=\left\{u \subseteq \omega_{1}:|u|=\omega\right\}$. We obtain a proof of the second theorem from our proof of the first by replacing $P$ everywhere by $Q$ and relativizing all neighborhoods to the subspace. The paper has been written so that all else remains intact (see the corollary in $\S 8$ ).

At the end of the paper we discuss more of the history of the subject and some open (and in some cases longstanding) questions.

Acknowledgments. The author thanks Amer Bešlagić and Scott Williams for independently checking the proof. The author also thanks the referee for a very careful and thoughtful report.

\section{Notation AND TERMinOLOGY}

Subspaces and neighborhoods. We will use $\omega_{1} \times \omega_{1}$ for the index set. For each $f: \omega_{1} \times \omega_{1} \rightarrow \omega+1$, let Fin $f$ be the restriction of $f$ to $\left\{\langle\alpha, \beta\rangle \in \omega_{1} \times \omega_{1}\right.$ : $f(\alpha, \beta)<\omega$ ) (so Fin $f$ is the part of $f$ that assumes finite values and is a partial function with respect to $\left.\omega_{1} \times \omega_{1}\right)$. For each $\delta \in \omega_{1}$, let $Y_{\delta}=\left\{f \in{ }^{\omega_{1} \times \omega_{1}} \omega+1: \exists \varepsilon \in\right.$ $\left.\omega_{1}\left[\operatorname{Dom} F i n f \subseteq(\{0\} \times \varepsilon) \cup\left(\delta \backslash\{0\} \times \omega_{1}\right)\right]\right\}$ (for a function $F$, Dom $F$ and $\operatorname{Ran} F$ denote the domain and range respectively). For each $\delta \in \omega_{1}$ and each $f \in Y_{\delta}$, let $l(f)$ be the least $\varepsilon$ satisfying the above condition: so $\forall \beta \geq l(f), f(0, \beta)=\omega$, and $\forall \beta<l(f) \exists \gamma \geq \beta[f(0, \gamma)<\omega]$. Note that $l(f)$ does not depend upon $\delta$. Let $X=\bigcup\left\{Y_{\delta}: \delta \in \omega_{1}\right\}$.

For each $f: \omega_{1} \times \omega_{1} \rightarrow \omega+1$ and each $g: \omega_{1} \times \omega_{1} \rightarrow \omega$ where $g$ extends Fin $f$, define the $g$-neighborhood of $f$ by $N(f, g)=\left\{h \in \omega_{1} \times \omega_{1} \omega+1\right.$ : Fin $h$ extends Fin $f$, and, $\left.\forall\langle\alpha, \beta\rangle \in \omega_{1} \times \omega_{1}, h(\alpha, \beta) \geq g(\alpha, \beta)\right\}$ (a function extends itself). Then $\operatorname{Ran} N$ is a base for the topology of $\square^{\omega_{1} \times \omega_{1}}(\omega+1)$, where each member is both open and closed (see Lemma 1). As a result of the extension property possessed by any function in a basic open set about $f$, we have that $X$ and each $Y_{\delta}$ (and any other set defined by constraining from above the domain of the finite part of the function) are closed in $\square^{\omega_{1} \times \omega_{1}}(\omega+1)$. We will use $\mathrm{Cl}$ to denote the closure operator for the box topology.

Extensions and membership with the exception of a single index. Fix $\left\langle\alpha_{0}, \beta_{0}\right\rangle \in \omega_{1} \times \omega_{1}$. Suppose $f, h: \omega_{1} \times \omega_{1} \rightarrow \omega+1$. Then Fin $h$ extends Fin $f$ off $\left\langle\alpha_{0}, \beta_{0}\right\rangle$ iff, for each $\langle\alpha, \beta\rangle \in$ Dom Fin $f$ with $\langle\alpha, \beta\rangle \neq\left\langle\alpha_{0}, \beta_{0}\right\rangle, h(\alpha, \beta)=f(\alpha, \beta)$.

Suppose $f: \omega_{1} \times \omega_{1} \rightarrow \omega+1$ and $g: \omega_{1} \times \omega_{1} \rightarrow \omega$ where $g$ extends Fin $f$. Then define $N(f, g) \mid\left\langle\alpha_{0}, \beta_{0}\right\rangle=\left\{h\right.$ : Fin $h$ extends Fin $f$ off $\left\langle\alpha_{0}, \beta_{0}\right\rangle$, and $\forall\langle\alpha, \beta\rangle \neq$ $\left.\left\langle\alpha_{0}, \beta_{0}\right\rangle, h(\alpha, \beta) \geq g(\alpha, \beta)\right\}$. 
Set-theoretical limit. For each $\mathcal{F} \subseteq \omega_{1} \times \omega_{1} \omega+1$, define $\Psi(\mathcal{F}): \omega_{1} \times \omega_{1} \rightarrow \omega+1$ by $\Psi(\mathcal{F})(\alpha, \beta)=\omega$ if $f(\alpha, \beta)=\omega$ for every $f \in \mathcal{F}$, and otherwise, $\Psi(\mathcal{F})(\alpha, \beta)=$ $\operatorname{Lub}\{f(\alpha, \beta): f \in \mathcal{F}$ and $\langle\alpha, \beta\rangle \in$ Dom Fin $f\}$ (of course this least upper bound may equal $\omega$ also).

A sequence $\left\langle f_{n}: n \in \omega\right\rangle$ in $X$ has a set-theoretical limit iff there is a fixed index $\left\langle\alpha_{0}, \beta_{0}\right\rangle$ such that for each $n \in \omega$, Fin $f_{n+1}$ extends Fin $f_{n}$ off $\left\langle\alpha_{0}, \beta_{0}\right\rangle$, and the sequence of functional values $\left\langle f_{n}\left(\alpha_{0}, \beta_{0}\right): n \in \omega\right\rangle$ is strictly increasing (and therefore) converging to $\omega$. The set-theoretical value of the limit is the function $\Psi(\operatorname{Ran} f)$; so the limit takes the functional value $\omega$ at $\left\langle\alpha_{0}, \beta_{0}\right\rangle$, the finite part of the limit extends the finite part of each term of the sequence off $\left\langle\alpha_{0}, \beta_{0}\right\rangle$, and the domain of the finite part of the limit is equal to $\bigcup\left\{\right.$ Dom Fin $\left.f_{n}: n \in \omega\right\} \backslash\left\{\left\langle\alpha_{0}, \beta_{0}\right\rangle\right\}$. (Note our use of the adjective set-theoretical to distinguish this concept from that of a limit point in the box topology.)

Ordinals. Subsets of $\omega_{1}$ inherit a well-ordering. We give $\omega_{1}$ the order topology and use interval notation for open, half-open, and closed intervals. Suppose $\Gamma \subseteq \omega_{1}$ (included is the special case $\Gamma=\gamma \in \omega_{1}$ ). With respect to the subspace topology, let $L(\Gamma)=\{\delta \in \Gamma: \delta$ is a limit point in $\Gamma\}$, and let $I(\Gamma)=\Gamma \backslash L(\Gamma)$ (i.e., the set of all isolated points). We assume the usual ordinal addition and multiplication (exponentiation, however, always refers to cardinals).

We will also need the following function. Let $\theta: \omega_{1} \rightarrow \omega_{1}$ be the unique function such that: (1) the restriction of $\theta$ to $L\left(\omega_{1}\right)$ is an order-isomorphism of $L\left(\omega_{1}\right)$ onto $\omega_{1} ;(2) \forall \gamma \in L\left(\omega_{1}\right),(\gamma, \gamma+\omega]$ is a point-inverse set; and $(3)[0, \omega]$ is a point-inverse set. (It follows of course that $\forall \gamma \in L\left(L\left(\omega_{1}\right)\right),\{\gamma\}$ is a point-inverse set.)

\section{Outline OF The PROOF}

Trees. The heart of our proof that the box product is nonnormal is the recursive construction of a tree $\langle T, \preceq\rangle$ (Theorems 3,4 , and 5 ) where the point-set $T$ is a closed and discrete subset of $X$ (Theorem 6).

As usual, we mean that $\preceq$ is transitive, reflexive, and antisymmetric, and for each $f \in T,\{g \in T: g \prec f\}$ is well ordered by $\prec$. For each $f \in T, H t(f)$ is the order type of $\{g \in T: g \prec f\}$; for each ordinal $\delta, \operatorname{Lev}_{\delta}(T)=\{f \in T: H t(f)=\delta\}$; and $H t(T)$ is the least $\delta$ with $\operatorname{Lev}_{\delta}(T)=\varnothing$. For each $f \in T$ and each $\delta \leq H t(f)$, let $\operatorname{Pred}_{\delta}(f)=g$ where $g \preceq f$ and $g \in \operatorname{Lev}_{\delta}(T)$.

A branch of $T$ is the range of a 1-1 order-preserving function $b: \nu \rightarrow T$ where $\nu \leq H t(T)$ and for every $\mu \in \nu, b(\mu) \in \operatorname{Lev}_{\mu}(T)$ (for convenience, we will also refer to the function $b$ as a branch of $T) ; b$ is maximal iff $b$ does not have a proper extension (for our construction below, this is equivalent to the nonexistence of an upper bound in $T$ for $\operatorname{Ran} b$ ).

Strategy. After constructing the tree, we then show that for every $E: T \rightarrow$ $\omega_{1} \times \omega_{1} \omega$ where for each $f, E(f)$ extends Fin $f$, there is an order-preserving sequence $\left\langle h_{n}: n \in \omega\right\rangle$ in $T$ (i.e., for each $n \in \omega, h_{n} \prec h_{n+1}$ ) possessing a set-theoretical limit such that each neighborhood of the limit intersects $N\left(h_{n}, E\left(h_{n}\right)\right)$ for all but finitely many $n$. This result (Theorem 7) and the fact that $T$ is closed and discrete prove the failure of paracompactness and also serve as an auxiliary lemma for the nonnormality proofs in $\S \S 7$ and 8 . The next step is to show that while $T$ can be separated, it is also possible to partition $T$ into two disjoint sets such that $\left\langle h_{n}: n \in \omega\right\rangle$ is forced to alternate between the two sets (Theorem 8). Since every 
subset of $T$ is closed, the failure of normality is a corollary. We give a second proof of nonnormality by showing that $T$ cannot be separated from every closed set in its complement. Let $T^{\prime}$ be the set-theoretical derived set of $T$; i.e., $T^{\prime}$ is the set of all set-theoretical limits of sequences of points in $T$. The set $T^{\prime}$ is also closed and discrete; and disjointness between $T$ and $T^{\prime}$ is built into the fact that each limit lives at the end of a maximal branch in $T$. In light of Theorem $7, T$ and $T^{\prime}$ cannot be separated which simultaneously proves the failure of normality and the collectionwise Hausdorff property (Main Theorem, $\S 8$ ).

Details. We now outline some of the properties of the tree that will enable us, given an open cover, to construct a corresponding sequence $\left\langle h_{n}: n \in \omega\right\rangle$ as described in the subsection on strategy. The height of the tree is $\omega_{1}$, each point has $2^{\omega_{1}}$ immediate successors, and every branch is countable. For each $f \in T, l(f) \in$ $L\left(\omega_{1}\right), f(0, \beta)<\omega$ for each $\beta<l(f)$, and $H t(f)=\theta(l(f))$; moreover, we define about $f$ an open-closed set $U(f)$ with the following properties. For all $f, g \in T$ : (1) if $f \neq g$, then $U(f)$ and $U(g)$ are either comparable (with respect to proper set inclusion) or disjoint; and (2) $U(g) \subseteq U(f)$ iff $f \preceq g$. Also, the intersection of each nested collection in $\operatorname{Ran} U$ is open. For each $f \in T$, the set of all immediate successors of $f$ has two key features which reflect the basic obstacles which must be overcome: the fact that we cannot determine in advance either the fixed index $\left\langle\alpha_{0}, \beta_{0}\right\rangle$ where $\left\langle h_{n}\left(\alpha_{0}, \beta_{0}\right): n \in \omega\right\rangle$ is increasing, or the open cover (defined by $E$ ).

Let $f, g \in T$ with $g$ an immediate successor of $f$. Then $l(g)=l(f)+\omega$, and there exists $\lambda<l(f)$ such that Fin $g$ extends Fin $f$ off $\langle 0, \lambda\rangle$, and $g(0, \lambda)=f(0, \lambda)+1$. Thus, each immediate successor looks below $\langle 0, l(f)\rangle$ to increase exactly one value. This process defines a regressive function $\rho(g)$ which extends $\rho(f)$. We impose on these functions a requirement of gap preservation: a regressive function $r$ is gap preserving provided that for all limit ordinals $\delta$ and $\varepsilon$ in Dom $r$ with $\delta<$ $\varepsilon, r(\varepsilon) \notin(r(\delta), \delta]$ (i.e., the half-open interval with endpoints $r(\delta)$ and $\delta$ ) (the special properties of these functions are described in Theorems 1 and 2). As a consequence of gap preservation, the set of indices on which an immediate successor is permitted to increase the value has the order type of a simple sequence and is cofinal in $\{\langle 0, \beta\rangle: \beta<l(f)\}$. The immediate successor set of $f$ is thereby partitioned into countably many levels according to the index on which the value is changed. So the relationship between each point of the tree and its predecessors is described by a regressive function; this is the first of the two features referred to above.

A branch of the tree terminates as soon as the value of one particular index has been increased an infinite number of times. Each function looking back to this particular index has an immediate predecessor. The sequence of predecessors, $\left\langle h_{n}: n \in \omega\right\rangle$, has a set-theoretical limit. Let $k$ be the value of the limit, and let $\left\langle\alpha_{0}, \beta_{0}\right\rangle$ be the fixed index on which the functions are strictly increasing (so $\left.\alpha_{0}=0\right)$. If, for each $n \in \omega, h_{n+1} \in \bigcap\left\{N\left(h_{m}, E\left(h_{m}\right)\right) \mid\left\langle\alpha_{0}, \beta_{0}\right\rangle: m \leq n\right\}$, then every neighborhood of $k$ intersects $N\left(h_{n}, E\left(h_{n}\right)\right)$ for all but finitely many $n$; the second feature (referred to above) guarantees that we can satisfy the antecedent of this conditional.

For each $f \in T$ and each immediate successor $g$, a function $A(g): \omega_{1} \times \omega_{1} \rightarrow \omega$ is defined so that $A(g)$ extends Fin $g$ and the following conditions are satisfied (for the clauses below, we will say that a function $G$ dominates a function $F$ provided that, at each argument, the value of $G$ is greater than or equal to the value of $F$ ): 
(1) Suppose $f \in T, \lambda<l(f)$, and $g$ is an immediate successor of $f$ which looks back to $\langle 0, \lambda\rangle$ to increase the value. Then for each of the finitely many predecessors $g_{0}$ of $f$ where $g_{0}$ looks back to either $\langle 0, \lambda\rangle$ or an allowable index preceding $\langle 0, \lambda\rangle$, $g$ dominates $A\left(g_{0}\right)$.

(2) Suppose $E$ is a function defining a basic open cover of $T$. Then for each $f \in T$, there is an immediate successor $g$ such that, $A(g)$ dominates $E(f)$ (this together with the fact that $A(g)$ extends Fin $g$ implies that $N(g, A(g)) \mid\langle 0, \lambda\rangle \subseteq$ $N(f, E(f)) \mid\langle 0, \lambda\rangle$, where $g$ looks back to $\langle 0, \lambda\rangle)$.

So the function $A$ allows us to code open sets with points (we chose $\omega_{1} \times \omega_{1}$ for the index set to facilitate the definition of $A$ ); and each open cover thereby determines a subordinate subtree (i.e., the set of all $j \in T$ such that, for each $f$ and $g$ with $f \prec g \preceq j$ and $g$ an immediate successor of $f, A(g)$ dominates $E(f))$. Every maximal branch of the subtree contains the range of a sequence $\left\langle h_{n}: n \in \omega\right\rangle$ with the required properties.

\section{REgRESSIVE FUNCTIONS}

Gap preserving regressive functions. For each $\gamma \in L\left(\omega_{1}\right)$ with $\gamma>\omega$, let $\operatorname{Reg}_{\gamma}$ be the set of all functions $r: L(\gamma) \rightarrow I(\gamma)$ such that:

(1) $\forall \delta \in L(\gamma), r(\delta)<\delta$;

(2) $\forall \delta, \varepsilon \in L(\gamma)$ with $\delta<\varepsilon, r(\varepsilon) \leq r(\delta)$ or $r(\varepsilon)>\delta$, i.e., $r(\varepsilon) \notin(r(\delta), \delta]$;

(3) $\forall \delta \in L(\gamma)$, each point inverse set of $r$ restricted to $\delta$ is finite.

By (1), $r$ is regressive. By (2), every time a gap is created we prohibit the mapping of larger arguments into the gap. By (3), a function in $\operatorname{Reg}_{\gamma}$ with an infinite pointinverse set does not have any proper extensions in $\operatorname{Reg}_{\delta}$ for $\delta>\gamma$ (since an infinite point-inverse set is necessarily cofinal in the domain).

For each $\gamma$ and each $r \in \operatorname{Reg}_{\gamma}$, let $\tau(r)=\{\delta \in I(\gamma): \forall \varepsilon \in L(\gamma)$ with $\varepsilon>$ $\delta, r(\varepsilon) \geq \delta$. This definition depends only upon $r$ since $\gamma=\varepsilon+\omega$ if $\varepsilon$ is the largest element in Dom $r$, and $\gamma=\operatorname{Lub} \operatorname{Dom} r$ if there is no largest element. Note that in the former case, $\tau(r)$ is always infinite; moreover, $\tau(r) \backslash \operatorname{Ran} r$ is infinite. Also note that every proper extension of $r$ maps the argument $\gamma$ into $\tau(r)$ and maps every point above $\gamma$ either into $\tau(r)$ or to some other point above $\gamma$.

If $\tau(r)$ is infinite, then let $\sigma(r): \omega \rightarrow \tau(r)$ be the (unique onto) order-isomorphism (see Theorem 2 below).

Theorem 1. Suppose $\gamma \in L\left(\omega_{1}\right)$ with $\gamma>\omega$, and $r \in \operatorname{Reg}_{\gamma}$. Then the following are equivalent:

(1) $\tau(r)$ is finite;

(2) there exists $\mu \in \operatorname{Ran} r$ such that $r^{-1}(\mu)$ is order-isomorphic to $\omega$, and for each $\nu \in \operatorname{Ran} r$ with $\nu \neq \mu, r^{-1}(\nu)$ is finite.

Theorem 2. Suppose $\gamma \in L\left(\omega_{1}\right)$ with $\gamma>\omega$, and $r \in \operatorname{Reg}_{\gamma}$. Then the following are equivalent:

(1) $\tau(r)$ is infinite;

(2) $\tau(r)$ is order-isomorphic to $\omega$ and cofinal in $\gamma$;

(3) each point-inverse set of $r$ is finite;

(4) for each $\delta \in L(\gamma),\{\varepsilon \in L(\gamma): \varepsilon \geq \delta$ and $r(\varepsilon)<\delta\}$ is finite.

Proof of Theorem 1. Suppose $\tau(r)$ is finite. We always have $\tau(r) \neq \varnothing$ since $0 \in$ $\tau(r)$. Let $\mu$ be the largest point in $\tau(r)$. The following argument, due to the referee, is a simplification of the original. First note the following fact: by condition (3) 
in the definition of Reg, no point-inverse set of $r$ has a limit point in $\gamma$. Let $\delta=\operatorname{Lub} r^{-1}(\mu)$. By the fact, either $\delta=\gamma$ or $\delta \in r^{-1}(\mu)$; by gap preservation, the choice of $\delta$, and $\mu \in \tau(r)$, the second case implies that $\delta+1 \in \tau(r)$ contradicting the choice of $\mu$. Hence $r^{-1}(\mu)$ is cofinal in $\gamma$ and order-isomorphic to $\omega$. Suppose $\nu<\mu$. Since $\mu \in \tau(r), \mu$ is an upper bound for $r^{-1}(\nu)$, so by the fact noted above, $r^{-1}(\nu)$ is finite. Suppose $\mu<\nu<\gamma$. Choose $\delta>\nu$ with $\delta \in r^{-1}(\mu)$. By gap preservation, $\delta$ is an upper bound for $r^{-1}(\nu)$, so as above, $r^{-1}(\nu)$ is finite.

Suppose that clause (2) in Theorem 1 holds and $\tau(r)$ is infinite. Let $\lambda$ be the limit of a strictly increasing sequence in $\tau(r)$. By the definition of $\tau$ and (1) in the definition of Reg, $\lambda \notin \operatorname{Dom} r$, and therefore, $\lambda=\gamma$. However, $r^{-1}(\mu)$ is cofinal in $\gamma$ forcing $\lambda \leq \mu<\gamma$.

Proof of Theorem 2. Suppose $\tau(r)$ is infinite. As we argued in the last paragraph in the proof of Theorem 1, the range of any strictly increasing sequence in $\tau(r)$ is cofinal in $\gamma$, and this of course proves the existence of an order-isomorphism defined on $\omega$ and the nonexistence of a cofinal point-inverse set; so we have proofs of $(1) \Rightarrow(2)$ and $(1) \Rightarrow(3)$. Since it is both illustrative and central to our application below, we give a characterization of the isomorphism using point-inverse sets.

Define recursively $\nu: \omega \rightarrow \tau(r)$ and $\lambda: \omega \rightarrow \gamma$ by: $\nu_{0}=0$ (the least point in $\tau(r)$ ), and $\lambda_{0}$ is the largest point in $r^{-1}\left(\nu_{0}\right)$ if $\nu_{0} \in \operatorname{Ran} r$, and $\lambda_{0}=\nu_{0}$ otherwise (existence of $\lambda_{0}$ in the first case follows from $\tau(r) \cap\left(\nu_{0}, \gamma\right) \neq \varnothing$ ); and for $n>0$, $\nu_{n}=\lambda_{n-1}+1$ (the least point in $\tau(r) \cap\left(\lambda_{n-1}, \gamma\right)$ ), and $\lambda_{n}$ is the largest point in $r^{-1}\left(\nu_{n}\right)$ if $\nu_{n} \in \operatorname{Ran} r$, and $\lambda_{n}=\nu_{n}$ otherwise (existence of $\lambda_{n}$ follows from $\left.\tau(r) \cap\left(\nu_{n}, \gamma\right) \neq \varnothing\right)$. As noted above, $\operatorname{Ran} \nu$ is cofinal in $\gamma$. By the minimality in the choice of $\nu, \operatorname{Ran} \nu=\tau(r)$, so $\nu$ is an order-isomorphism. We also have explicit bounds on the point-inverse sets since for each $n \in \omega$ and each $\xi \in \operatorname{Ran} r \cap$ $\left[\nu_{n}, \lambda_{n}\right], r^{-1}(\xi) \subseteq\left[\nu_{n}, \lambda_{n}\right]$.

For the remaining implications, $(2) \Rightarrow(1)$ is immediate, and $(3) \Rightarrow(1)$ follows from Theorem $1 ;(4) \Rightarrow(3)$ is also immediate.

Suppose (4) fails and choose $\delta \in L(\gamma)$ and $\varepsilon: \omega \rightarrow L(\gamma)$ such that $\varepsilon$ is strictly increasing and for each $n \in \omega, \varepsilon_{n} \geq \delta$ while $r\left(\varepsilon_{n}\right)<\delta$. Then by gap preservation, for each $n \in \omega, r\left(\varepsilon_{n+1}\right) \leq r\left(\varepsilon_{n}\right)$; so $r$ has an infinite point-inverse set causing (3) to fail.

\section{Construction of the tree}

Auxiliary functions. We now define $\left\langle\operatorname{Lev}_{\delta}(T): \delta \in \omega_{1}\right\rangle$ and the partial order by recursion on $\delta$. The definition requires four recursively defined auxiliary functions: $\rho, A, M$, and $S$. The limit case uses the restriction of $\rho$ on levels below $\delta$, and the successor case uses the restrictions of both $\rho$ and $A$. Also for the successor case, $M$ and $S$ are defined explicitly in terms of $\rho$ and $A$. We ultimately obtain:

(1) $\rho(f) \in \operatorname{Reg}_{l(f)}$ (for $l(f)>\omega$ ), and $\operatorname{Dom} \rho=T \cup\{b: b$ is a branch in $T\}$ (the definition on $T$ is extended to each branch by taking unions);

(2) $A(g): \omega_{1} \times \omega_{1} \rightarrow \omega$ where $A(g)$ is an extension of Fin $g$, and $\operatorname{Dom} A=$ $\bigcup\left\{\operatorname{Lev}_{\delta}(T): \delta\right.$ is a successor ordinal $\}$ (so $g$ is an immediate successor of some $f$ );

(3) $M(f): \omega_{1} \times \omega_{1} \rightarrow{ }^{\omega} \omega$, and $\operatorname{Dom} M=T$; and

(4) $S(f): \omega \times P \rightarrow X$, and $\operatorname{Dom} S=T$ (recall that $P=\left\{u \subseteq \omega_{1}:|u|=\omega_{1}\right\}$ ).

We define the set of all immediate successors of $f$ to be $\operatorname{Ran} S(f)$. The two key features of the immediate successor set of $f$ discussed in the outline of the proof are reflected in the two arguments of the function $S(f)$. 
Coding open sets. The following notation will be used in the definitions of $S$ and $A$ to code open sets. Well order each of $P$ and $P \times 2^{\omega_{1}}$ in type $2^{\omega_{1}}$, and by recursion define $t^{\prime}: P \rightarrow P \times 2^{\omega_{1}}$ by letting $t^{\prime}(u)$ be the least $\langle v, \alpha\rangle$ such that $u \subseteq v$ and $\langle v, \alpha\rangle$ does not belong to the range of $t^{\prime}$ restricted to the predecessors of $u$ (note that $t^{\prime}$ is a 1-1 correspondence). Let $t$ be the composition of $t^{\prime}$ followed by projection to the first coordinate.

Then $t: P \rightarrow P$ such that:

(1) $\forall u \in P, u \subseteq t(u)$; and

(2) $\forall v \in P,\left|t^{-1}(v)\right|=2^{\omega_{1}}$.

We now partition each point-inverse set of $t$ into $c$ many parts so that each part has cardinality $2^{\omega_{1}}$. Let $s: P \rightarrow{ }^{\omega} \omega$ such that, for each $\xi \in{ }^{\omega} \omega$ and each $v \in P$, $\left|s^{-1}(\xi) \cap t^{-1}(v)\right|=2^{\omega_{1}}$. Let $B: P \rightarrow{ }^{\omega_{1} \times \omega_{1}} \omega$ such that, for each $\xi \in{ }^{\omega} \omega$ and each $v \in P$, the restriction of $B$ to $s^{-1}(\xi) \cap t^{-1}(v)$ is a 1-1 correspondence onto ${ }^{\omega_{1} \times \omega_{1}} \omega$.

Choice of a root. Let $r t: \omega_{1} \times \omega_{1} \rightarrow \omega+1$ be defined by $r t(\alpha, \beta)=0$ if $\alpha=0$ and $\beta<\omega$, and $r t(\alpha, \beta)=\omega$ otherwise. Let $\operatorname{Lev}_{0}(T)=\{r t\}$; and let $\rho(r t)$ be the empty function.

Successor case. Suppose $\delta \in \omega_{1}$ and for every $\varepsilon \leq \delta, \operatorname{Lev}_{\varepsilon}(T) \subseteq X$ has been constructed, along with the appropriate restrictions of $\prec, \rho$ and $A$, so that for each $h \in \operatorname{Lev}_{\varepsilon}(T):$

(1) $\varepsilon=\theta(l(h))$ and $l(h) \in L\left(\omega_{1}\right)$;

(2) $\rho(h) \in \operatorname{Reg}_{l(h)}($ for $h \neq r t)$; and,

(3) $\tau(\rho(h))$ is infinite (for $h \neq r t)$.

Suppose $f \in \operatorname{Lev}_{\delta}(T)$, and let $r=\rho(f)$.

(1) Definition of $M(f)$. Suppose $\delta=0$ (in which case, $f=r t$ ). Then for each $\langle\alpha, \beta\rangle \in \omega_{1} \times \omega_{1}$ and each $n \in \omega$, let $M(f)(\alpha, \beta)(n)=n$.

Suppose $\delta>0$. For each $n \in \omega$, let $\Delta_{n}=\{\sigma(r)(m): m \leq n\}$. By Theorem 2, for each $n \in \omega, r^{-1}\left[\Delta_{n}\right]$ is finite. For each $\langle\alpha, \beta\rangle \in \omega_{1} \times \omega_{1}$, let $M(f)(\alpha, \beta): \omega \rightarrow \omega$ be a strictly increasing sequence, such that, for each $n \in \omega, M(f)(\alpha, \beta)(n) \geq$ $\operatorname{Max}\left\{A(g)(\alpha, \beta): \exists \gamma \in r^{-1}\left[\Delta_{n}\right]\left[g=\operatorname{Pred}_{\theta(\gamma)+1}(f)\right]\right\}$. Note that, for each $\gamma \in$ $L\left(\omega_{1}\right), \theta(\gamma+\omega)=\theta(\gamma)+1$; so if $l(f)=\gamma+\omega$, then $\operatorname{Pred}_{\theta(\gamma)+1}(f)=f$.

(2) Definition of $S(f)$. Suppose $\langle n, u\rangle \in \omega \times P$. For each $i \in \omega$, let $\varepsilon_{i}=l(f)+i+1$. Let $\lambda=\sigma(r)(n)$ if $f \neq r t$, and let $\lambda=n$ otherwise. Let $S(f)(n, u)=g$ where $g$ is defined by the following clauses.

We need two cases. Suppose $\delta \geq \omega$. Then:

(2.0) $g(0, l(f))=M(f)(0, l(f))(n)$;

(2.1) $g(0, \lambda)=f(0, \lambda)+1$;

(2.2) $\forall \beta<l(f)$ with $\beta \neq \lambda, g(0, \beta)=f(0, \beta)$;

(2.3) $\forall i \in \omega, g\left(0, \varepsilon_{i}\right)=\operatorname{Max}\left\{M(f)\left(0, \varepsilon_{i}\right)(n), s(u)(i)\right\}$;

(2.4) $\forall \beta>l(f)$ with $\beta \notin \operatorname{Ran} \varepsilon, g(0, \beta)=\omega$;

(2.5) $\forall\langle\alpha, \beta\rangle$ with $0<\alpha<\delta, g(\alpha, \beta)=f(\alpha, \beta)$;

(2.6) $\forall \beta \in u, g(\delta, \beta)=M(f)(\delta, \beta)(n)$;

(2.7) $\forall \beta \in \omega_{1} \backslash u, g(\delta, \beta)=\omega$; and

(2.8) $\forall\langle\alpha, \beta\rangle$ with $\alpha>\delta, g(\alpha, \beta)=\omega$.

Suppose $\delta<\omega$. Then in clauses (2.5) through (2.8), replace $\delta$ with $\delta+1$. All else remains intact.

Let $\operatorname{Lev}_{\delta+1}(T)=\bigcup\left\{\operatorname{Ran} S(h): h \in \operatorname{Lev}_{\delta}(T)\right\}$. 
(3) Definition of $\rho$. For $g=S(f)(n, u)$, let $\rho(g)$ be the one point extension of $r(=\rho(f))$ where $\rho(g)(\gamma)=\sigma(r)(n)$ for $\gamma=l(f)$ and $l(f)>\omega$, and $\rho(g)(\gamma)=n$ for $\gamma=l(f)=\omega$ (so the extension depends only upon the first argument).

(4) Definition of $A$. For $g=S(f)(n, u)$, let $A(g): \omega_{1} \times \omega_{1} \rightarrow \omega$ be the extension of Fin $g$ such that, for each $\langle\alpha, \beta\rangle \in \omega_{1} \times \omega_{1} \backslash$ Dom Fin $g, A(g)(\alpha, \beta)=B(u)(\alpha, \beta)$ (so in this case, the extension depends only upon the second argument).

Limit case. Suppose $\delta \in L\left(\omega_{1}\right)$ and for every $\varepsilon<\delta, \operatorname{Lev}_{\varepsilon}(T)$ has been constructed, along with the restrictions of $\prec$ and $\rho$, so that $\rho$ is order-preserving with respect to $\prec$ in the domain and proper extension in the range.

For every branch $b: \delta \rightarrow T$, extend $\rho$ (using the recursion hypothesis) by $\rho(b)=$ $\bigcup\{\rho(b(\varepsilon)): \varepsilon \in \delta\}$, and let $\Psi(b)$ abbreviate $\Psi(\operatorname{Ran} b)(\Psi$ is defined in $\S 1)$.

Let $\operatorname{Lev}_{\delta}(T)=\{\Psi(b) \mid b: \delta \rightarrow T$ is a branch of $T$ with $\tau(\rho(b))$ infinite $\}$. For each branch $b: \delta \rightarrow T$ where $\tau(\rho(b))$ is infinite, let $\rho(\Psi(b))=\rho(b)$, and for each $\varepsilon \in \delta$, let $\operatorname{Pred}_{\varepsilon}(\Psi(b))=b(\varepsilon)$.

Partition of the successor set. Suppose $n \in \omega$ and $f, g \in T$ with $f \prec g$. Let $h=\operatorname{Pred}_{\delta+1}(g)$ where $\delta=H t(f)$. Then $g$ is a level $n$ successor of $f$ iff, for some $u \in P, h=S(f)(n, u)$. Thus every successor is classified by the level of the immediate successor through which it passes. If $g$ is a level $n$ successor of $f$, then $g(0, l(f))=M(f)(0, l(f))(n)$ (see Theorem 4 below). This fact will be used in the proof that $T$ is closed and discrete.

Also note that this partition defines an order-preserving function from $T$ into the partial functions from $\omega_{1}$ into $\omega$ partially ordered by extension: for each $f \in T$, send $f$ to $\tilde{f}$ where $\operatorname{Dom} \tilde{f}=H t(f)$ and for each $\alpha \in \operatorname{Dom} \tilde{f}, \tilde{f}(\alpha)=n$ provided that $f$ is a level $n$ successor of $\operatorname{Pred}_{\alpha}(f)$.

Theorem 3. The ordered pair $\langle T, \preceq\rangle$ is a tree possessing the following properties (these results follow immediately from the definitions):

(1) $H t(T)=\omega_{1}$

(2) for every $f \in T$ :

(2.1) $H t(f)=\theta(l(f))$ and $l(f) \in L\left(\omega_{1}\right)$;

(2.2) $\rho(f) \in \operatorname{Reg}_{l(f)}$ and $\tau(\rho(f))$ is infinite $($ for $f \neq r t)$; and

(2.3) $f$ has $2^{\omega_{1}}$ immediate successors in $T$;

(3) for each $\delta \in \omega_{1}, \operatorname{Lev}_{\delta}(T) \subseteq Y_{\varepsilon} \backslash \bigcup\left\{Y_{\gamma}: \gamma<\varepsilon\right\}$ where $\varepsilon=\delta$ if $\delta \geq \omega$, and $\varepsilon=\delta+1$ otherwise; and

(4) if $\delta \in L\left(\omega_{1}\right)$ and $f, g \in \operatorname{Lev}_{\delta}(T)$ such that $\{h \in T: h \prec f\}=\{h \in T: h \prec g\}$, then $f=g$.

Theorem 4. Suppose $f, g \in T, n \in \omega$, and $g$ is a level $n$ successor of $f$. Let $\lambda$ be the strictly increasing sequence of ordinals defined by $\lambda=\sigma(\rho(f))$. Then:

(1) $\rho(g)$ extends $\rho(f)$;

(2) for each $\beta \in l(f) \backslash\left\{\lambda_{0}, \ldots, \lambda_{n}\right\}, g(0, \beta)=f(0, \beta)<\omega$;

(3) for each $\beta \in\left\{\lambda_{0}, \ldots, \lambda_{n}\right\}, f(0, \beta)<\omega$ and $g(0, \beta)=f(0, \beta)+m$ where $m<\omega$ and is the cardinality of $\{\gamma \geq l(f): \rho(g)(\gamma)=\beta\}$ (in particular, $g\left(0, \lambda_{n}\right)>$ $\left.f\left(0, \lambda_{n}\right)\right) ;$ and

(4) $g(0, l(f))=M(f)(0, l(f))(n)$.

Proof. The proof is by induction on $g$ with $f$ and $n$ held fixed. The first clause is immediate from the definitions. We assume (as an induction hypothesis) that $f(0, \beta)<\omega$ for each $\beta<l(f)$; it then follows by clauses (2) and (3) of the theorem, 
and (2.0), (2.3) in the definition of $S$, that $g(0, \beta)<\omega$ for each $\beta<l(g)$. We sketch the induction arguments for clauses (2) and (3) leaving the obvious induction hypothesis implicit. The successor case for (2) follows from $\rho(g)(l(f))=\lambda_{n}$, together with gap preservation and (2.2) in the definition of $S$. The limit case is immediate from the definition of $\Psi$. The successor case for (3) follows from (2.1) and (2.2) in the definition of $S$. For the limit case, each point-inverse set of $\rho(g)$ is finite by Theorem 2 and (2.2) in Theorem 3, so as above, the result follows from the definition of $\Psi$.

To prove the fourth clause, let $\delta=H t(f)$ and let $h=\operatorname{Pred}_{\delta+1}(g)$. By the second clause with $h$ in the role of $f, g(0, l(f))=h(0, l(f))$ since $l(f) \in L\left(\omega_{1}\right)$ while $\tau(\rho(h)) \subseteq I\left(\omega_{1}\right)$; in turn, $h(0, l(f))=M(f)(0, l(f))(n)$ by $(2.0)$ in the definition of $S$.

Theorem 5. Suppose $b: \nu \rightarrow T$ is a branch of $T$. Then:

(1) $b$ is maximal iff $\tau(\rho(b))$ is finite;

(2) $\nu<\omega_{1}$ (i.e., every branch is countable); and

(3) if $\nu$ is a successor ordinal, then $\Psi(b)=b(\nu-1)$.

Proof. The first property is by the definition of $\operatorname{Lev}_{\delta}(T)$ for $\delta \in L\left(\omega_{1}\right)$. The second follows from the first, Theorem 4(1), the fact that a regressive function defined on $L\left(\omega_{1}\right)$ has at least one infinite point-inverse set, and Theorem 1 . The third clause follows from Theorem 4 and (2.5) in the definition of $S$.

Remark. Suppose $b: \nu \rightarrow T$ is a branch of $T$. Then $\rho(b)$ has an infinite point-inverse set (and $b$ is therefore maximal) iff there exist $n \in \omega$ and a strictly increasing cofinal sequence $\varepsilon: \omega \rightarrow \nu$ such that, for each $m \in \omega, b\left(\varepsilon_{m}+1\right)$ is a level $n$ successor of $b\left(\varepsilon_{m}\right)$; so a branch terminates as soon as for a fixed value of $n$, the branch passes through an infinite number of immediate level $n$ successors. We can simultaneously have cofinal sequences as described above for more than one value of $n$, in which case, the least such value determines the ordinal with the infinite point-inverse set.

\section{Closed And Discrete POINT-SET}

Theorem 6. The point-set of the tree $\langle T, \preceq\rangle$ is closed and discrete.

(A proof is contained in Lemmas 1-7.)

Open-closed sets. For each $f \in T$, let $U(f)$ be the set of all $g: \omega_{1} \times \omega_{1} \rightarrow \omega+1$ such that:

(1) $\forall\langle\alpha, \beta\rangle \in$ Dom Fin $f$ with $\alpha>0, g(\alpha, \beta)=f(\alpha, \beta)$;

(2) $\forall\langle\alpha, \beta\rangle \notin$ Dom Fin $f$ with $\omega \leq \alpha<H t(f), g(\alpha, \beta) \geq M(h)(\alpha, \beta)(n)+1$ where $h=\operatorname{Pred}_{\alpha}(f)$ and $f$ is a level $n$ successor of $h$; and for $\alpha$ satisfying $0 \leq \alpha<$ $\operatorname{Min}\{\omega, H t(f)\}, g(\alpha+1, \beta) \geq M(h)(\alpha+1, \beta)(n)+1$; and

(3) $\{\beta<l(f): g(0, \beta) \neq f(0, \beta)\}$ is a finite subset of $I\left(\omega_{1}\right)$.

For each $f \in T$, let $V(f)=\{g \in U(f): \forall \beta<l(f), g(0, \beta) \geq f(0, \beta)\}$. Note that $f \in V(f) \subseteq U(f)$.

Lemma 1. Basic neighborhoods are closed; for all $f \in T$, each of $U(f)$ and $V(f)$ is both open and closed.

Proof. Let $j$ be the partial function defined by the lower bound in condition (2) of the definition of $U$. Then $U(f)=\bigcup\{N(h, k): h \in U(f)$ and $\forall\langle\alpha, \beta\rangle \in$ $\operatorname{Dom} j, k(\alpha, \beta) \geq j(\alpha, \beta)\} ;$ and $V(f)=\bigcup\{N(h, k): h \in V(f) ; \forall\langle\alpha, \beta\rangle \in \operatorname{Dom} j$, $k(\alpha, \beta) \geq j(\alpha, \beta) ; \forall \beta<l(f), k(0, \beta) \geq f(0, \beta)\}$; so $U(f)$ and $V(f)$ are open. 
Each of $N(f, g), U(f)$, and $V(f)$ is closed since $h \notin N(f, g)$ (resp., $U(f), V(f)$ ) implies that $N(h, k)$ is disjoint from $N(f, g)$ (resp., $U(f), V(f))$ where, for each $\langle\alpha, \beta\rangle \in$ Dom Fin $f \backslash$ Dom Fin $h, k(\alpha, \beta)=f(\alpha, \beta)+1$.

Lemma 2. Suppose $f, g \in T$. Then the following are equivalent:

(1) $f \preceq g$;

(2) for each $\langle\alpha, \beta\rangle$ with $0<\alpha<\delta, g(\alpha, \beta)=f(\alpha, \beta)$, where $\delta=H t(f)$ if $H t(f) \geq \omega$, and $\delta=H t(f)+1$ otherwise;

(3) $g \in U(f)$;

(4) $U(g) \subseteq U(f)$.

Moreover, if $f \neq g$, then $U(f)$ and $U(g)$ are either comparable (with respect to proper set inclusion) or disjoint.

Proof. For $(1) \Rightarrow(2)$, see the definitions of $S$ (clause (2.5)) and $\Psi$. For $(2) \Rightarrow(1)$, suppose (2) holds. By Theorem 3(3), $H t(f) \leq H t(g)$; and by Theorem 3(4), $\{h \in$ $T: h \preceq f$ and $h \preceq g$ \} has a largest element $k$. We claim that $H t(f)=H t(k)$ forcing $f=k$. Otherwise, there exist $\langle n, u\rangle,\langle m, v\rangle \in \omega \times P$ with $\operatorname{Pred}_{\alpha+1}(f)=S(k)(n, u)$ and $\operatorname{Pred}_{\alpha+1}(g)=S(k)(m, v)$ where $\alpha=H t(k)$; in turn, our hypothesis forces $n=m$ and $u=v$, and this contradicts the choice of $k$.

For $(1) \Rightarrow(3)$, the first two conditions for membership in $U(f)$ follow from $(1) \Leftrightarrow(2)$, and the third from Theorem 4. To prove the converse, define $k$ and $\alpha$ as in the first paragraph. Then either $H t(f)=H t(k)$, or $\operatorname{Pred}_{\alpha+1}(g)=\operatorname{Pred}_{\alpha+1}(f)$ (by (2.6) and (2.7) in the definition of $S$ and (1) and (2) in the definition of $U$ ) and as above this contradicts the choice of $k$.

If $U(g) \subseteq U(f)$, then $g \in U(f)$ is immediate. If $g \in U(f)$, then by the equivalence of conditions (1) through (3), on those indices $\langle\alpha, \beta\rangle$, with $\alpha>0$, constrained by both $U(f)$ and $U(g)$, there is agreement; and for $\alpha=0$, satisfaction of condition (3) for $U(g)$ implies satisfaction of the same condition for $U(f)$ (by Theorem 4, again).

In light of the equivalence of conditions (1) through (4), the addendum follows from $U(f) \cap U(g)=\varnothing$ whenever $f$ and $g$ are distinct immediate successors of the same point $(S-(2.6),(2.7)$ and $U-(1),(2))$.

Lemma 3. Suppose $b: \nu \rightarrow T$ is a branch of $T$. Then $\bigcap\{U(b(\mu)): \mu \in \nu\}$ is open.

Proof. If $g$ satisfies $U(f)-(3)$, then each point in $\{h$ : Fin $h$ extends Fin $g\}$ satisfies the condition also. For the first two conditions, whenever two of the open sets both place a constraint on an index, their values coincide (as we first noted in the proof of Lemma 2).

Lemma 4. Suppose $h \in \mathrm{Cl}(T)$. Then $\{f \in T: h \in U(f)\}$ is a branch of $T$ with a largest element.

Proof. Since $T \subseteq U(r t)$ (Lemma 2) and $U(r t)$ is closed (Lemma 1), $h \in U(r t)$. The sets in Ran $U$ are either comparable or disjoint (Lemma 2), so we can define a branch $b: \nu \rightarrow T$ with $\operatorname{Ran} b=\{f \in T: h \in U(f)\}$. The intersection $\bigcap\{U(b(\mu)): \mu \in \nu\}$ is an open set (Lemma 3) containing $h$. If $b$ is maximal, then the intersection is disjoint from $T$ (Lemma 2 again) contradicting $h \in \mathrm{Cl}(T)$; so $\Psi(b) \in T$. If $\nu$ is a limit ordinal, then $h \in \bigcap\{U(b(\mu)): \mu \in \nu\} \backslash U(\Psi(b))$ which is also an open set disjoint from $T$ (we are using Theorem 3(4) in addition to the preceding lemmas), again contradicting $h \in \mathrm{Cl}(T)$.

Lemma 5. Suppose $f \in T$ and $h \in U(f)$ with $h(0, l(f))=\omega$. Then $h \notin \mathrm{Cl}(\bigcup\{V(j)$ : $j \in T$ with $j \succ f\}$ ). 
Proof. First note that if $j \in T$ with $j \succ f$, then each point in $V(j)$ agrees with $j$ on $\langle 0, l(f)\rangle$; this follows from the restriction to $I\left(\omega_{1}\right)$ in the third clause of the definition of $U$ and $V(j) \subseteq U(j)$.

Let $\lambda=\sigma(\rho(f))$. Then $\lambda$ is a 1-1 sequence where, for each $n \in \omega, \lambda_{n}<l(f)$. By the third clause in the definition of $U$ specifying finite disagreement, we can choose $m \in \omega$ so that, for each $n \geq m, h\left(0, \lambda_{n}\right)=f\left(0, \lambda_{n}\right)$. Let $k: \omega_{1} \times \omega_{1} \rightarrow \omega$ be an extension of Fin $h$ where $k(0, l(f))=M(f)(0, l(f))(m)$. Then by the fourth clause of Theorem 4 for each $j \in T$ with $j \succ f$, the inequality $j(0, l(f)) \geq k(0, l(f))$ implies that $j$ is a level $n$ successor of $f$ for some $n \geq m$; and in turn, $j\left(0, \lambda_{n}\right)>f\left(0, \lambda_{n}\right)$, also by Theorem 4; so by the definition of $V, N(h, k) \cap V(j)=\varnothing$.

Lemma 6. For each $f \in T, \bigcup\{V(j): j \in T$ with $j \succ f\}$ is closed.

Proof. Let $h \in \mathrm{Cl}(\bigcup\{V(j): j \succ f\})$. Since the closure is contained in $U(f)$ (by Lemmas 1 and 2 and $V(j) \subseteq U(j)), h(0, l(f)) \neq \omega$ by Lemma 5 . By the fourth clause of Theorem 4, and the restriction to $I\left(\omega_{1}\right)$ in the third clause of the definition of $U$, there exists $n \in \omega$ with $h(0, l(f))=M(f)(0, l(f))(n)$. Let $k: \omega_{1} \times \omega_{1} \rightarrow \omega$ be an extension of Fin $h$ such that, for each $\langle\alpha, \beta\rangle \notin$ Dom Fin $h$, $k(\alpha, \beta) \geq M(f)(\alpha, \beta)(n)+1$. Let $\delta=H t(f)$ if $H t(f) \geq \omega$ and let $\delta=H t(f)+1$ otherwise; and let $u=\left\{\beta \in \omega_{1}: h(\delta, \beta)=M(f)(\delta, \beta)(n)\right\}$. Then either $u \in P$ and $h \in V(g)$ where $g=S(f)(n, u)$, or there is an open set $\mathcal{O}$ such that $h \in \mathcal{O} \subseteq N(h, k)$ and $\mathcal{O} \cap \bigcup\{V(j): j \succ f\}=\varnothing$ contradicting the choice of $h$ (let $\mathcal{O}=N(h, k)$ if $u \notin P$; otherwise, impose an additional requirement on $k$ : for each $\beta<l(g)$ with $h(0, \beta)=\omega, k(0, \beta) \geq g(0, \beta)+1)$.

Lemma 7. Suppose $f \in T$ and $h \in U(f) \backslash \bigcup\{U(j): j \in T$ with $j \succ f\}$. Then $h$ is isolated from $T$.

Proof. Let $\mathcal{O}=U(f) \backslash \bigcup\{V(j): j \succ f\}$. Then: (1) $\mathcal{O}$ is an open set by Lemmas 1 and $6 ;(2) h \in \mathcal{O}$ by the hypothesis and the inclusion $V(j) \subseteq U(j)$; and, (3) $\mathcal{O} \cap T=\{f\}$ by Lemma 2 and $j \in V(j)$.

\section{Subordination of a branch to AN OPEN COVER}

Fundamental open cover. Suppose that, for each $f \in T, E(f): \omega_{1} \times \omega_{1} \rightarrow \omega$ is an extension of Fin $f$. Then define

$$
\mathcal{C}(E) \text { by } \mathcal{C}(E)=\{N(f, E(f)): f \in T\} \cup\{\operatorname{Com} T\}
$$

where $\operatorname{Com} T$ is the complement of $T$ in the box product. We will refer to $\mathcal{C}(E)$ as a fundamental open cover.

Subordination. Let $\mathcal{C}=\mathcal{C}(E)$ be a fundamental open cover. A branch $b: \nu \rightarrow T$ is subordinate to $\mathcal{C}$ provided that for each $\mu \in \nu$ (with $\mu<\nu-1$ if $\nu$ is a successor ordinal), the following condition is satisfied. Let $f=b(\mu)$ and let $g=b(\mu+1)$. Then for each $\langle\alpha, \beta\rangle \in \omega_{1} \times \omega_{1}, A(g)(\alpha, \beta) \geq E(f)(\alpha, \beta)$ (i.e., $A(g)$ dominates $E(f))$.

Canonical sequence. Suppose $b: \nu \rightarrow T$ is a maximal branch of $T$. Define the canonical sequence for $b,\left\langle h_{n}: n \in \omega\right\rangle$, by $h_{n}=b\left(\theta\left(\gamma_{n}\right)\right)$ where $\gamma$ is the orderisomorphism of $\omega$ onto the unique infinite point-inverse set of $\rho(b)$ (see Theorems 1 and 5). A canonical sequence belongs to an open cover $\mathcal{C}=\mathcal{C}(E)$ if it corresponds to a maximal branch of $T$ which is, in addition, subordinate to $\mathcal{C}$. Note that 
maximality only refers to $T$ (i.e., first take a maximal branch of $T$ and then check for subordination), so an existence proof is needed.

Theorem 7. Suppose $\mathcal{C}$ is a fundamental open cover. Then there exist canonical sequences belonging to $\mathcal{C}$, and each of these sequences has a set-theoretical limit at the value of which $\mathcal{C}$ fails to be locally finite.

(A proof is contained in Lemmas 8-10.)

Lemma 8. Suppose $\mathcal{C}=\mathcal{C}(E)$ is a fundamental open cover, and $b: \nu \rightarrow T$ is a nonmaximal branch. Suppose further that $b$ is subordinate to $\mathcal{C}$. Then $b$ has a proper extension which is subordinate to $\mathcal{C}$; moreover, if $\nu$ is a successor ordinal and $f=b(\nu-1)$, then we can define the extension of $b$ at $\nu$ to be a level $n$ successor of $f$ for all but finitely many $n$.

Proof. Suppose $\nu$ is a limit ordinal. Then there is only one extension: $b(\nu)=\Psi(b)$ (the left-hand refers to the extension and the right-hand refers to the original). Subordination in this case is automatic since the definition only mentions immediate successors.

Suppose $\nu$ is a successor ordinal and let $f=b(\nu-1)$. Let $\delta=\nu-1$ if $\nu \geq \omega$, and let $\delta=\nu$ if $\nu<\omega$. Let $m \in \omega$ such that $m \geq E(f)(0, l(f))$ and $E(f)^{-1}([0, m]) \cap$ $\left(\{\delta\} \times \omega_{1}\right)$ has cardinality $\omega_{1}$. Let $v \in P$ with $v \subseteq\left\{\beta \in \omega_{1}: E(f)(\delta, \beta) \leq m\right\}$; and let $\xi \in{ }^{\omega} \omega$ such that, for each $i \in \omega, \xi(i) \geq E(f)\left(0, \varepsilon_{i}\right)$ where $\varepsilon_{i}=l(f)+i+1$.

Let $n \in \omega$ with $n \geq m$ (recall that, for all $\langle\alpha, \beta\rangle, M(f)(\alpha, \beta)(n) \geq n)$. By the choice of $B$ we can choose $u \in s^{-1}(\xi) \cap t^{-1}(v)$ such that $B(u)=E(f)$. (Note that while each depends upon $m$, the choices of $n$ and $u$ are otherwise independent of one another.) Let $b(\nu)=S(f)(n, u)$. Subordination holds by the definitions of $S$ and $A$.

Lemma 9. Every canonical sequence has a set-theoretical limit: Suppose $b \in \nu \rightarrow$ $T$ is a maximal branch. Let $\left\langle h_{n}: n \in \omega\right\rangle$ be the canonical sequence corresponding to $b$, and let $\lambda$ be the unique point in $\operatorname{Ran} \rho(b)$ with an infinite point-inverse set. Then for each $n \in \omega$, Fin $h_{n+1}$ extends Fin $h_{n}$ off $\langle 0, \lambda\rangle$, and $\left\langle h_{n}(0, \lambda): n \in \omega\right\rangle$ is strictly increasing converging to $\omega$; it follows that $\left\langle h_{n}: n \in \omega\right\rangle$ has a set-theoretical limit, and that the value of the limit is $\Psi(b)$.

Proof. Let $\gamma$ be the order-isomorphism of $\omega$ onto $\rho(b)^{-1}(\lambda)$, and for each $n \in \omega$, let $g_{n}$ be the immediate successor of $h_{n}$ in $\operatorname{Ran} b$. Then by the definition of $S$, we have that for each $n \in \omega$ :

(1) $l\left(h_{n}\right)=\gamma_{n}$;

(2) $l\left(g_{n}\right)=\gamma_{n}+\omega$

(3) $\rho\left(g_{n}\right)\left(\gamma_{n}\right)=\lambda$;

(4) Fin $g_{n}$ extends Fin $h_{n}$ off $\langle 0, \lambda\rangle$; and

(5) $g_{n}(0, \lambda)=h_{n}(0, \lambda)+1$.

We also have, by gap preservation and Theorem 4, that for each $n \in \omega$ : for each $j \in \operatorname{Ran} b$ with $g_{n} \preceq j \prec g_{n+1}$, and each $\beta<l\left(h_{n}\right), j(0, \beta)=g_{n}(0, \beta)$. So by taking $j=h_{n+1}$, it follows that Fin $h_{n+1}$ extends Fin $h_{n}$ off $\langle 0, \lambda\rangle$ (we are using Lemma 2 for indices $\langle\alpha, \beta\rangle$ where $\alpha>0)$ and $h_{n+1}(0, \lambda)=h_{n}(0, \lambda)+1$.

Lemma 10. Suppose $\mathcal{C}=\mathcal{C}(E)$ is a fundamental open cover, and $b: \nu \rightarrow T$ is a maximal branch of $T$. Suppose further that $b$ is subordinate to $\mathcal{C}$. Let $\left\langle h_{n}: n \in \omega\right\rangle$ be the canonical sequence corresponding to $b$, and let $k$ be the set-theoretical limit. Then every open set about $k$ intersects $N\left(h_{n}, E\left(h_{n}\right)\right)$ for all but finitely many $n$. 
Proof. Define the ordinal $\lambda$ and the sequence $g$ as in Lemma 9. First note that by the nature of a set-theoretical limit, if $k \in \bigcap\left\{N\left(h_{n}, E\left(h_{n}\right)\right) \mid\langle 0, \lambda\rangle: n \in \omega\right\}$, then every neighborhood of $k$ intersects $N\left(h_{n}, E\left(h_{n}\right)\right)$ for all but finitely many $n$. This in turn will follow if we can show that, for each $n \in \omega, h_{n+1} \in \bigcap\left\{N\left(h_{m}, E\left(h_{m}\right)\right) \mid\langle 0, \lambda\rangle\right.$ : $m \leq n\}$ (again by the nature of a limit). The keystone of the argument is the choice of $M$ and its role as a lower bound in the definition of $S$.

Suppose $m, n \in \omega$ and $j \in \operatorname{Ran} b$ with $m \leq n$ and $g_{n} \preceq j \prec g_{n+1}$. Then $j$ dominates $A\left(g_{m}\right)$ (i.e., $\forall\langle\alpha, \beta\rangle \in \omega_{1} \times \omega_{1}, j(\alpha, \beta) \geq A\left(g_{m}\right)(\alpha, \beta)$ ); so by subordination, $j$ also dominates $E\left(h_{m}\right)$. This follows from Theorem 4 and Lemma 2 for $\langle\alpha, \beta\rangle \in$ Dom Fin $g_{m}$, and from gap preservation and the role of $M$ in the definition of $S$ for $\langle\alpha, \beta\rangle \notin$ Dom Fin $g_{m}$. This result with $j=h_{n+1}$ together with the fact that Fin $h_{n+1}$ extends Fin $h_{m}$ off $\langle 0, \lambda\rangle$ (Lemma 9) implies that $h_{n+1} \in N\left(h_{m}, E\left(h_{m}\right)\right) \mid\langle 0, \lambda\rangle$.

\section{Partition of the Point-Set of the tree}

Theorem 8. The point-set $T$ can be separated by a pairwise disjoint collection of open sets; however, there is a function $\chi: T \rightarrow\{0,1\}$ such that the closures of each pair of open sets separating $\chi^{-1}(0)$ and $\chi^{-1}(1)$ have a common point of intersection.

(A proof is contained in Lemmas 11-14.)

Corollary (to Theorems 6 and 8). The box product of $\omega_{1}$ many copies of $\omega+1$ is nonnormal.

Lemma 11. The set $T$ can be separated.

Proof. For each $h \in T$, let $W(h)=\{k \in U(h)$ : Fin $k$ extends Fin $h\}$; so Ran $W$ is a collection of basic neighborhoods. We claim that $\{W(h): h \in T\}$ is pairwise disjoint. By Lemma 2 and the inclusion $W(h) \subseteq U(h)$, we need only consider the case $f \prec g$. By Theorem 4, there exists $\lambda<l(f)$ such that $\langle 0, \lambda\rangle \in$ Dom Fin $f \cap$ Dom Fin $g$ and $f(0, \lambda) \neq g(0, \lambda)$, so by the extension condition for membership in basic neighborhoods, $W(f) \cap W(g)=\varnothing$.

Lemma 12. Suppose $\chi: T \rightarrow\{0,1\}$ such that every fundamental open cover has a canonical sequence where the terms alternate between $\chi^{-1}(0)$ and $\chi^{-1}(1)$. Then the closures of each pair of open sets separating $\chi^{-1}(0)$ and $\chi^{-1}(1)$ have a common point of intersection.

(This follows immediately from Lemma 10.)

Partition of $T$. Define $\chi: T \rightarrow\{0,1\}$ recursively as follows: $\chi(r t)=0$, and for each $\delta \in \omega_{1} \backslash\{0\}$, and each $f \in \operatorname{Lev}_{\delta}(T), \chi(f)=0$ iff $\left\{\lambda \in \tau(\rho(f)): \rho(f)^{-1}(\lambda)=\varnothing\right.$, or $\chi(g)=1$ where $\gamma$ is the largest element in $\rho(f)^{-1}(\lambda)$ and $\left.g=\operatorname{Pred}_{\theta(\gamma)}(f)\right\}$ is infinite. Note that for each $\delta \in I\left(\omega_{1}\right)$ and each $f \in \operatorname{Lev}_{\delta}(T), \chi(f)=0$.

Lemma 13. Suppose that $b: \nu \rightarrow T$ is a nonmaximal branch of $T$ such that $\tau(\rho(b)) \backslash \operatorname{Ran} \rho(b)$ is finite. Let $\lambda$ be the order-isomorphism of $\omega$ onto $\tau(\rho(b)) \cap$ Ran $\rho(b)$. For each $n \in \omega$, let $\gamma_{n}$ be the largest element in $\rho(b)^{-1}\left(\lambda_{n}\right)$, and let $g_{n}=b\left(\theta\left(\gamma_{n}\right)\right)$. Then $\left\{n \in \omega: \chi\left(g_{n}\right) \neq \chi(\Psi(b))\right\}$ is infinite.

(This follows immediately from the recursion defining $\chi$.) 
Alternating condition. Suppose $b: \nu \rightarrow T$ is a nonmaximal branch of $T$. Then $b$ is alternating provided that, for each $\lambda \in \operatorname{Ran} \rho(b)$, the following is satisfied: Let $\left\langle\gamma_{0}, \ldots, \gamma_{n}\right\rangle$ be an order-preserving enumeration of $\rho(b)^{-1}(\lambda)$ (where $n \in \omega$ ). Then for each $m \leq n, \chi\left(b\left(\theta\left(\gamma_{m}\right)\right)\right)=0$ iff $m$ is even.

Lemma 14. Suppose $\mathcal{C}$ is a fundamental open cover, and $b: \nu \rightarrow T$ is a nonmaximal branch of $T$ which is subordinate to $\mathcal{C}$ and alternating. Then $b$ can be extended while preserving each of these two conditions.

Proof. If $\nu$ is a limit ordinal, then the extension $b(\nu)=\Psi(b)$ satisfies automatically both subordination and alternation. Suppose $\nu$ is a successor ordinal and let $f=$ $b(\nu-1)$ and let $r=\rho(f)$. Note that whether an extension of $f$ preserves the alternating condition depends only upon the corresponding extension of $r$ and its placement of $l(f)$ in an appropriate point-inverse set (in particular, the value of $\chi$ on the extension is immaterial); so we are only concerned with the level of the extension of $f$. By Lemma 8 , we can choose $m \in \omega$ so that, for every $n \geq m$, there is a level $n$ successor of $f$ which preserves subordination. If $\tau(r) \backslash \operatorname{Ran} r$ is infinite, then choose $n \geq m$ with $\sigma(r)(n) \notin \operatorname{Ran} r$. Otherwise, by Lemma 13, we can choose $n \geq m$ so that $\chi(f) \neq \chi(g)$ where:

(1) $\lambda=\sigma(r)(n)$

(2) $\lambda \in \operatorname{Ran} r$;

(3) $\gamma$ is the largest element in $r^{-1}(\lambda)$; and

(4) $g=\operatorname{Pred}_{\theta(\gamma)}(f)$.

\section{Closed discrete sets Which CAnnot Be Separated}

Main Theorem. The box product of $\omega_{1}$ many copies of $\omega+1$ is neither normal nor collectionwise Hausdorff.

Proof. Let $T^{\prime}=\{\Psi(b): b$ is a maximal branch of $T\}$; so (by Lemma 9) $T^{\prime}$ is the set of all set-theoretical limits of sequences in $T$. We claim that the union $T \cup T^{\prime}$ is a closed and discrete subset of $X$. The Main Theorem follows immediately from the claim and Theorem 7 (of course, $T$ and $T^{\prime}$ are the two sets which cannot be separated).

In light of Theorem 6 , to prove the claim we need only show that $T^{\prime}$ is closed and discrete.

Extend the function $W$ (defined in the proof of Lemma 11) to $T \cup T^{\prime}$ by defining $W(\Psi(b))=\bigcap\{U(b(\mu)): \mu \in \nu\}$ for each $b$ where $b: \nu \rightarrow T$ is a maximal branch of $T$. By Lemmas 1 and 3,W( $\Psi(b))$ is an open-closed set; and by Lemma 2 and Theorem $4, \Psi(b) \in W(\Psi(b))$. By Lemma 2, the range of $W$ restricted to $T^{\prime}$ is pairwise disjoint; so we have in one step that $T^{\prime}$ is discrete and can be separated. (Thus, while failing to separate the union, $\operatorname{Ran} W$ contains subcollections separating $T$ and $T^{\prime}$ individually, and each set in Ran $W$ intersects $T \cup T^{\prime}$ in exactly one point ( $T$-sets miss $T^{\prime}$ by Theorem 4, and $T^{\prime}$-sets miss $T$ by Lemma 2).)

For the proof that $T^{\prime}$ is closed, first note that, for each branch $b: \nu \rightarrow T, \Psi(b) \in$ $\bigcap\{V(b(\mu)): \mu \in \nu\}$ (again by Lemma 2 and Theorem 4).

Let $h \in X \backslash T^{\prime}$. We will use several cases to prove the existence of an open set $\mathcal{O}$ with $h \in \mathcal{O}$ and $\mathcal{O} \cap T^{\prime}=\varnothing$. If $h \notin U(r t)$, then let $\mathcal{O}=\operatorname{Com} U(r t)$ (i.e., the complement of $U(r t)$ in the box product) (see Lemmas 1 and 2).

Assume $h \in U(r t)$. Then there is a branch $b: \nu \rightarrow T$ such that $\operatorname{Ran} b=\{f \in$ $T: h \in U(f)\}$ (Lemma 2). 
Case 1. Suppose $b$ is maximal. Then let $\mathcal{O}=W(\Psi(b)) \cap \operatorname{Com}\{\Psi(b)\}$.

Case 2. Suppose $b$ is nonmaximal and $\nu \in L\left(\omega_{1}\right)$. Then let

$$
\mathcal{O}=\bigcap\{U(b(\mu)): \mu \in \nu\} \backslash U(\Psi(b)) .
$$

Case 3. Suppose $\nu \in I\left(\omega_{1}\right)$. Then let $f=b(\nu-1)(\nu$ cannot be 0$)$ and let $\mathcal{O}=U(f) \backslash \bigcup\{V(j): j \succ f\}$ (see Lemmas 1 and 6 ).

Corollary (to the paper). Suppose $c=2^{\omega_{1}}$. Then the closed subspace

$$
\left\{f \in \square^{\omega_{1} \times \omega_{1}}(\omega+1): \mid \text { Fin } f \mid \leq \omega\right\}
$$

is neither normal nor collectionwise Hausdorff.

Proof. First note that the machinery we put in place to allow us to construct maximal branches subordinate to a given open cover (see the definition of $P$ and the choices of $s, t$, and $B$ ) simply required the same number of points as open sets. Recall that $Q=\left\{u \subseteq \omega_{1}:|u|=\omega\right\}$. Throughout the paper, replace each occurrence of $P$ with $Q$ and each neighborhood with the intersection of the neighborhood and the subspace. The basic concepts affected are the choices of $s, t$, and $B$, and the definitions of $A$ and $S$; the set-theoretic hypothesis is now needed for the pointinverse requirement on $s$ and $t$. With these changes, all of the above theorems, lemmas, and proofs remain intact; moreover, we now have that $\mid$ Fin $f \mid \leq \omega$ for each $f \in T \cup T^{\prime}$.

\section{Stone's PRoblem (For Metrizable faCtor SPACES)}

Countable index set. Suppose $\Lambda$ is a separable metric space. As we noted in the introduction, if $\Lambda$ is locally compact and CH holds, then $\square^{\omega}(\Lambda)$ is paracompact (Rudin, 1972, $\left[\mathrm{Ru}_{1}\right]$ ); but if we take $\Lambda$ to be the irrationals, then $\square^{\omega}(\Lambda)$ is nonnormal in ZFC (E. K. van Douwen, 1975, $\left.\left[\mathrm{vD}_{1}\right]\right)$. However, local compactness in $\Lambda$ is not a necessary condition for paracompactness in the box product: $\mathrm{CH}$ implies that $\square^{\omega}$ (rationals) is paracompact (Lawrence, 1988, $\left[\mathrm{L}_{1}\right]$ ). These results raise several questions. (In addition to the papers cited above, see $\left[\mathrm{vD}_{2}, \mathrm{Ku}_{2}, \mathrm{Mil}, \mathrm{Ro}_{1}, \mathrm{Ro}_{2}\right.$, $\mathrm{Wil}_{1}, \mathrm{Wil}_{2}, \mathrm{Win}_{1}, \mathrm{Win}_{2}$ ] for the main results on normality and paracompactness in $\square^{\omega}(\Lambda)$; and see $\left[\mathrm{L}_{2}\right]$ for a recent survey.)

(1) A standard theorem is the identification of the irrationals with ${ }^{\omega} \omega$ with the product topology. Kenneth Kunen pointed out to the author that if $\Lambda \subseteq{ }^{\omega} \omega$ is eventually dominant and has the subspace topology, then $\square^{\omega}(\Lambda)$ is nonnormal in ZFC (a subset $D \subseteq{ }^{\omega} \omega$ is eventually dominant provided that $\forall f \in{ }^{\omega} \omega \exists g \in D \exists m \in$ $\omega \forall n \geq m[g(n) \geq f(n)])$; the proof uses Kunen's theorems on dominant families in $\left[\mathrm{Ku}_{1}\right]$. Is there a sufficient condition on a subspace of the irrationals weaker than domination (i.e., the subspace does not contain any closed isomorphic copies of a dominant family) so that the box product is nonnormal? For every subspace $\Lambda$ of the irrationals, $\Lambda \times \square^{\omega}(\omega+1)$ is nonnormal in ZFC iff $\Lambda$ is homeomorphic to an eventually dominant subspace (L. Wingers, $\left[\mathrm{Win}_{2}\right]$, first appearing as a preprint in 1992).

(2) Find a necessary and sufficient condition on $\Lambda$ so that $\square^{\omega}(\Lambda)$ is paracompact (normal) in ZFC $+\mathrm{CH}$. If $\Lambda$ is $\sigma$-compact and zero-dimensional, then $\square^{\omega}(\Lambda)$ is paracompact in $\mathrm{ZFC}+\mathrm{CH}$ (Wingers, [Win 1 ], first appearing as a preprint in 1991).

(3) Based on Rudin's paper $\left[\mathrm{Ru}_{1}\right]$ and results of E. A. Michael on abstract paracompact spaces $\left(1953,\left[\mathrm{Mic}_{1}\right]\right.$; 1957, $\left.\left[\mathrm{Mic}_{2}\right]\right)$, Kunen developed a theoretical framework for paracompactness in the box product for the case of a locally compact 
factor space $\left(1978,\left[\mathrm{Ku}_{2}\right]\right)$. Develop a theory in the absence of local compactness. One result might be an answer to Kunen's question: Does normality in $\square^{\omega}(\Lambda)$ imply paracompactness?

(4) (Rudin) Is paracompactness (normality) in $\square^{\omega}(\omega+1)$ independent of ZFC? This is undoubtedly the most important open question concerning box products.

Uncountable index set. Let $\Sigma$ be the closed subspace of $\square^{\omega_{1}}(\omega+1)$ consisting of all functions which assume the value $\omega$ on all but countably many indices.

(1) Is normality in $\Sigma$ independent of ZFC? From this paper, $c=2^{\omega_{1}}$ implies $\Sigma$ is nonnormal. If paracompactness holds in every finite subproduct of a given box product of arbitrarily many Hausdorff (but not necessarily metrizable) factor spaces, then for any point $f$ in the box product, the closed subspace $\{g: g$ disagrees with $f$ at most a finite number of times $\}$ is also paracompact (P. Nyikos and L. Piatkiewicz, [NyPi], first appearing as a preprint in 1993) (note that this result is in ZFC).

(2) (Williams) In particular, does $\mathrm{CH}$ imply that $\Sigma$ is paracompact? (Note that $\mathrm{CH}$ prevents the coding of open sets with points by the method of this paper; recall that this was one of the two key features of the immediate successor set of each point in our tree.)

(3) (Williams) Is $\square^{\omega_{1}}(\omega+1)$ countably paracompact (i.e., does every countable open cover have an open locally finite covering refinement)?

\section{REFERENCES}

$\left[\mathrm{vD}_{1}\right] \quad$ E. K. van Douwen, The box product of countably many metrizable spaces need not be normal, Fund. Math. 88 (1975), 127-132. MR 52:6640

$\left[\mathrm{vD}_{2}\right] \quad$, Covering and separation properties of box products, Surveys in General Topology, Academic Press, 1980, pp. 55-130. MR 81i:54006

[Kn] C. J. Knight, Box topologies, Quart. J. Math. 15 (1964), 41-54. MR 28:3398

$\left[\mathrm{Ku}_{1}\right] \quad \mathrm{K}$. Kunen, Some comments on box products, Colloq. Math. Soc. János Bolyai 10 (1975), 1011-1016. MR 52:15339

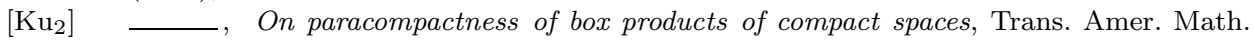
Soc. 240 (1978), 307-316. MR 58:24165

$\left[\mathrm{L}_{1}\right] \quad$ L. B. Lawrence, The box product of countably many copies of the rationals is consistently paracompact, Trans. Amer. Math. Soc. 309 (1988), 787-796. MR 89k:54053

$\left[\mathrm{L}_{2}\right] \quad$, Toward a theory of normality and paracompactness in box products, Ann. New York Acad. Sci. 705 (1993), 78-91. MR 95a:54038

[Mic 1 E. A. Michael, A note on paracompact spaces, Proc. Amer. Math. Soc. 4 (1953), 831-838. MR 15:144b

[Mic 2$] \quad$, Another note on paracompact spaces, Proc. Amer. Math. Soc. 8 (1957), 822828. MR 19:299c

[Mil] A. W. Miller, On box products Topology Appl. 14 (1982), 313-317. MR 84a:54014

[NyPi] P. Nyikos and L. Piatkiewicz, Paracompact subspaces in the box product topology, Proc. Amer. Math. Soc. (to appear).

[Ro 1 J. Roitman, Paracompact box products in forcing extensions, Fund. Math. 102 (1978), 219-228. MR 80j:03078

[Ro 2$] \quad$, More paracompact box products, Proc. Amer. Math. Soc. 74 (1979), 171-176. MR 80i:54006

$\left[\mathrm{Ru}_{1}\right] \quad \mathrm{M}$. E. Rudin, The box product of countably many compact metric spaces, General Topology and Appl. 2 (1972), 293-298. MR 48:2969

$\left[\mathrm{Ru}_{2}\right] \quad$, Lectures on set-theoretic topology, C.B.M.S. Regional Conf. Ser. in Math., no. 23, Amer. Math. Soc., Providence, RI, 1975. MR 51:4128

[Wil $\left.{ }_{1}\right] \quad$ S. W. Williams, Is $\square^{\omega}(\omega+1)$ paracompact? Topology Proc. 1 (1976), 141-146. MR 58: 18347 
[Wil 2 ] B Box products, Handbook of Set-Theoretic Topology, (K. Kunen and J. Vaughan, eds.), Elsevier, 1984, pp. 169-200. MR 87a:54007

[Win 1 L. Wingers, Box products of $\sigma$-compact spaces, Topology Appl. 56 (1994), 185-197. MR 94m:54026

[Win 2$] \_$, Box products and Hurewicz spaces, Topology Appl. (to appear).

Department of Mathematics, George Mason University, Fairfax, Virginia 22030-4444 